\title{
NONLOCAL VIBRATION ANALYSIS OF FLUID CONVEYING SINGLE-WALLED CARBON NANOTUBE WITH MAGNETIC EFFECTS
}

\author{
B. Ravi Kumar \\ School of Mechanical Engineering, SASTRA University, Thanjavur-613401, Tamilnadu, INDIA \\ E-mail: ravikumar@mech.sastra.edu
}

\begin{abstract}
In this work differential transform method (DTM) is used to study the vibration behavior of fluid conveying singlewalled carbon nanotube (SWCNT). Using the theories of thermo- elastic mechanics and nonlocal elasticity, a Euler Bernoulli beam model is developed for nonlocal vibration of a fluid conveying single-walled carbon nanotube resting on an elastic medium. The critical fluid velocity is being found out with different boundary conditions, i.e. Fixed-Fixed and simply supported at ends. Effects of nonlocal parameters, different elastic modulus and different magnetic field force on natural frequency and critical fluid velocity are being discussed. The differential transform method is used for the nonlocal vibration analysis, which yields to the results with high precision.
\end{abstract}

Keywords: Vibration, DTM, nonlocal, SWCNT, critical flow velocity.

(C) RASĀYAN. All rights reserved

\section{INTRODUCTION}

Nanoscale engineering materials have superior mechanical, electrical and thermal performances than the conventional structural materials. They have pulled in incredible enthusiasm for present day science and innovation after the development of carbon nanotubes by Iijima. ${ }^{1}$ Carbon nanotubes are allotropes of carbon with a round and hollow nanostructure. Nanotube has a length-to-diameter proportion of up to $132,000,000: 1$, fundamentally bigger than any other material. ${ }^{2}$ The structure of an SWCNT can be visualized by packaging a single-atom-thick layer of graphite called graphene into a flawless cylinder., SWCNTs are the perfect candidate for miniaturizing electronics rather than the micro- electromechanical scale currently used in electronics. ${ }^{6}$ SWCNTs are an important variety because they display electric properties that are not shared by the multi-walled carbon nanotube variants. ${ }^{7}$ CNTs are the strongest and stiffest materials in terms of tensile strength and elastic modulus respectively. ${ }^{8}$

The nonlocal continuum theory was proposed by Eringen ${ }^{4}$ for the analysis of small sized structures. In this theory, the effects of the small-scale are accounted by assuming that the stress at any point is a function of the strain at that point and the strains at all other points of the field. The mechanical analyses of nanostructures, theoretical and mathematical modeling becomes important issues when nanoengineering comes into the picture. This is due to the scale effect of the nanostructures. The influences of long range inter-atomic and intermolecular cohesive forces on the static and dynamic properties become significant as the length scales are reduced and cannot be neglected. The classical theory of elasticity does not include these effects because of the long-wave limit of the atomic theory and thus this theory would be unsuccessful to evaluate the structures with small-scale effects accurately. That is why the sizedependent continuum-based techniques are becoming more well-liked in analyzing these nanostructures as it provides much faster and accurate results. ${ }^{9-11}$

Sudak did the buckling analysis of multi-walled carbon nanotubes. ${ }^{9}$ Wang and Varadhan studied the small scale effect of carbon nanotube and shell model. ${ }^{10}$ Yakobson et al. developed an atomistic model for axially compressed SWCNT and did the comparison with a simple continuum shell model. ${ }^{11}$ Sears and Batra proposed a comprehensive buckling analysis of single walled and multi-walled carbon nanotubes 
using molecular mechanics simulations and continuum mechanics models. ${ }^{12}$ Reddy carried out bending, buckling and vibration analysis of nano beams using nonlocal theories. ${ }^{13}$

In the present work, the investigation of the vibration response of a fluid conveying single-walled carbon nanotube embedded in an elastic medium is carried out using the differential transformation method. Zhou ${ }^{16}$ proposed differential transformation method to solve both linear and non-linear initial value problems in electric circuit analysis. Later Chen and $\mathrm{Ho}^{17}$ applied this method to eigen value problems. Arikoglu and ozkol ${ }^{18}$ applied differential transformation method to solve the intergro-differential equation.

\section{EXPERIMENTAL}

The differential transform method is a semi-analytical method based on the Taylor series expansion. The differential transformation of the $\mathrm{i}^{\text {th }}$ derivative of the function $\mathrm{u}(\mathrm{x})$ is defined as follows:

$U(\mathrm{x})=\frac{1}{\mathrm{i} !}\left[\frac{d^{i} u(x)}{d x^{i}}\right]_{x=x_{0}}$

And the differential inverse transformation of $\mathrm{U}(\mathrm{i})$ is expressed as:

$u(x)=\sum_{i=0}^{\infty} U(\mathrm{i})\left(x-x_{0}\right)^{i}$

In real application function, $\mathrm{u}(\mathrm{x})$ is expressed as finite series and eq.-2 can be written as:

$u(x)=\sum_{i=0}^{n} U(\mathrm{i})\left(x-x_{0}\right)^{i}$

With the use of certain transformation rules, we can convert the governing differential equation and associated boundary conditions into some algebraic equations. These algebraic equations give a solution which provides the desired solution with high accuracy. Following transformation, the table is used for this purpose.

Table 1: Differential Transformations for Mathematical Equations

\begin{tabular}{|c|c|}
\hline Original Function & Transformed Function \\
\hline \multicolumn{2}{|c|}{$y(x)=u(x) \pm v(x) \quad Y(\mathrm{i})=U(\mathrm{i}) \pm V(\mathrm{i})$} \\
\hline$y(x)=\lambda u(x)$ & $Y(\mathrm{i})=\lambda U(\mathrm{i})$ \\
\hline$y(x)=\frac{d^{n} u(x)}{d x^{n}}$ & $Y(\mathrm{i})=(\mathrm{i}+1)(\mathrm{i}+2) \ldots(\mathrm{i}+n) U(\mathrm{i}+n)$ \\
\hline
\end{tabular}

\section{Formulation}

\section{A. Non-Local Formulation of SWCNT}

Eringen, first introduced nonlocal elasticity theory ${ }^{4}$, the simplified constitutive relation in a differential form is given as follows:

$$
\left(1-\left(e_{0} a\right)^{2} \nabla^{2}\right) \sigma=\tau
$$

Where $\tau$ is the classical macroscopic stress tensor at a point, a is the internal characteristic length (e.g., lattice parameter, coarse size, the length of carbon-carbon bonds), $\mathrm{e}_{0} \mathrm{a}$ is a material constant, $\sigma$ is nonlocal stress tensor and $\nabla^{2}$ is the Laplacian operator.

The nonlocal behavior can be considered negligible in the thickness direction for a beam structure. Thus, for a homogeneous isotropic beam, the nonlocal constitutive relation can be written as ${ }^{22}$

$\sigma_{x x}-\left(e_{0} a\right)^{2} \cdot \frac{\partial^{2} \sigma_{x x}}{\partial x^{2}}=E \varepsilon_{x x}$

Where $\mathrm{E}$ is the modulus of elasticity.

On the basis of the thermo- elastic mechanics theory, the axial force $\mathrm{N}_{\mathrm{t} 1}$ can be given as: ${ }^{22}$ 


$$
N_{t 1}=\frac{-E A}{1-2 v} \cdot \alpha_{x} T
$$

Where $\alpha_{\mathrm{x}}$ is the thermal expansion coefficient towards the direction of $\mathrm{x}$-axis and $v$ denotes the Poisson's ratio and $\mathrm{T}$ is the temperature change.

Lorentz magnetic force in axial direction is given by: ${ }^{14}$

$$
N_{m}=\mu A H_{X}^{2}
$$

Figure-1 shows the analysis model of the problem. $\mathrm{U}$ is the fluid velocity, $\mathrm{K}$ is the elastic constant for Winkler foundation and $\mathrm{L}$ is the length of the nanotube.

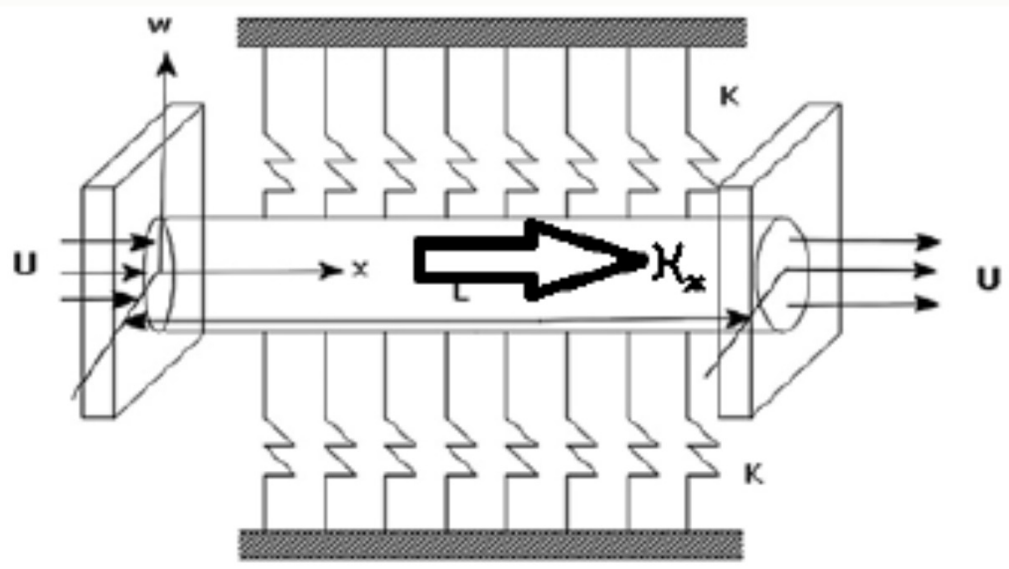

Fig.-1: Analysis model of CNT embedded in the elastic medium.

If we consider the thermal and magnetic effects, the partial differential equation of motion related to shear force of fluid conveying single-walled carbon nanotube is given by ${ }^{22}$,

$$
\frac{\partial Q}{\partial x}=m \frac{\partial^{2} w}{\partial t^{2}}-\frac{\partial\left(N_{t 1} \frac{\partial w}{\partial x}\right)}{\partial x}-\frac{\partial\left(N_{m} \frac{\partial w}{\partial x}\right)}{\partial x}+K w+M\left(\frac{\partial^{2} w}{\partial t^{2}}+2 U \frac{\partial^{2} w}{\partial x \partial t}+U^{2} \frac{\partial^{2} w}{\partial x^{2}}\right)
$$

Where $\mathrm{Q}$ is the shear force, $\mathrm{w}$ is the transverse deflection. $\mathrm{m}, \mathrm{M}$ denote the mass of nanotube per unit length and fluid mass per unit length of the beam respectively.

Take for convenience $\mathrm{N}_{\mathrm{t}}=\mathrm{N}_{\mathrm{t} 1}+\mathrm{N}_{\mathrm{m}}$.

Q satisfies the condition for equilibrium of Euler's beam $Q=\frac{\partial M_{1}}{\partial x}$

$$
M_{1}-\left(e_{0} a\right)^{2} \frac{\partial^{2} M_{1}}{\partial x^{2}}=-E I \frac{\partial^{2} w}{\partial x^{2}}
$$

Where $\mathrm{M}_{1}$ is the nonlocal bending moment and eq.-10 represents the nonlocal bending moment equation of nanotube.

By combining eqs.-8, 9 and 10, we have,

$$
M_{1}=-E I \frac{\partial^{2} w}{\partial x^{2}}+\left(e_{0} a\right)^{2}\left[m \frac{\partial^{2} w}{\partial x^{2}}-\frac{\partial\left(N_{t 1} \frac{\partial w}{\partial x}\right)}{\partial x}+K w+M\left(\frac{\partial^{2} w}{\partial t^{2}}+2 U \frac{\partial^{2} w}{\partial x \partial t}+U^{2} \frac{\partial^{2} w}{\partial x^{2}}\right)\right]
$$

Finally, by above equation and by eq.-10, the governing differential equation is given as: 


$$
\begin{aligned}
& {\left[E I+\left(e_{0} a\right)^{2} N_{t 1}-\left(e_{0} a\right)^{2} M U^{2}\right] \frac{\partial^{4} w}{\partial x^{4}}-2\left(e_{0} a\right)^{2} M U \frac{\partial^{4} w}{\partial x^{4}}-\left(e_{0} a\right)^{2}(M+m) \frac{\partial^{4} w}{\partial x^{2} \partial t^{2}}} \\
& +\left[M U^{2}-N_{t 1}-\left(e_{0} a\right)^{2} K\right] \frac{\partial^{2} w}{\partial x^{2}}+2 M U \frac{\partial^{2} w}{\partial x \partial t}+(M+m) \frac{\partial^{2} w}{\partial t^{2}}+K w=0
\end{aligned}
$$

In this study, theEuler-Bernoulli beam model using stress gradient approach for the dynamic analysis of single-walled carbon nanotube with nonlocal effect is considered. Where, $\mathrm{w}=\mathrm{w}(\mathrm{x}, \mathrm{t})$ denotes the beam deflection in the transverse direction, $\mathrm{x}, \mathrm{t}$ are the space coordinates, and $\mathrm{I}$ is the moment of inertia of carbon nanotube.

The eq. -12 can be non-dimensionalized by using $\mathrm{L}$ (length of carbon nanotube) and by substituting, W= $\mathrm{w} / \mathrm{L}$ and $\mathrm{X}=\mathrm{x} / \mathrm{L}$, we have;

$$
\frac{A}{L^{3}} \cdot \frac{d^{4} W}{d X^{4}}-B(i \omega) \frac{d^{3} W}{d X^{3}}-C \frac{(i \omega)^{2}}{L} \frac{d^{2} W}{d X^{2}}+\frac{D}{L} \cdot \frac{d^{2} W}{d X^{2}}+2 M U(i \omega) \frac{d W}{d X}+(M+m)(i \omega)^{2} L W+K L W=0
$$

Where,

$$
\begin{aligned}
& A=\left[E I+\left(e_{0} a\right)^{2} N_{t 1}-\left(e_{0} a\right)^{2} M U^{2}\right] \\
& B=2\left(e_{0} a\right)^{2} M U ; \\
& C=\left(e_{0} a\right)^{2}(M+m) \\
& D=\left[M U^{2}-N_{t 1}-\left(e_{0} a\right)^{2} K\right]
\end{aligned}
$$

\section{B. DTM Formulation}

In converting the governing equation in DTM formulation, we use Table-1 and we can conveniently write the following expression.

\section{Application of Boundary Conditions}

$$
W(\mathrm{i}+4)=\left[\begin{array}{l}
\left(B(i \omega) L \cdot(\mathrm{i}+1)(\mathrm{i}+2)(\mathrm{i}+3) W(\mathrm{i}+3)+C(i \omega)^{2} L^{2} \cdot(\mathrm{i}+1)(\mathrm{i}+2) W(\mathrm{i}+2)-D L^{2} \cdot(\mathrm{i}+1)\right. \\
(\mathrm{i}+2) \cdot W(\mathrm{i}+2)-2 M U(\mathrm{i} \omega) L^{3} \cdot(\mathrm{i}+1) W(\mathrm{i}+1)-(M+m)(i \omega)^{2} L^{4} \cdot W(\mathrm{i})+k L^{4} . \\
W(\mathrm{i})) /(\mathrm{A} \cdot(\mathrm{i}+1)(\mathrm{i}+2)(\mathrm{i}+3)(\mathrm{i}+4))
\end{array}\right]
$$

\section{Simply Supported at Both Ends}

The boundary conditions for the case of simply supported SWCNT at both the ends are as:

$$
w(0)=0, w^{\prime \prime}(0)=0, w(L)=0, w^{\prime \prime}(L)=0
$$

By using Differential Transformation these can be written as:

$$
\begin{aligned}
& W(0)=0, W(2)=\frac{M U\left(e_{0} a\right)^{2}(i \omega) L . W(1)}{E I+\left(e_{0} a\right)^{2} N_{t}} \\
& \sum_{i=0}^{N} W(\mathrm{i})=0, \sum_{i=0}^{N}\left[F . \mathrm{i}(\mathrm{i}-1)+2 M U\left(e_{0} a\right)^{2}(i \omega) L . \mathrm{i}+H\right] . W(\mathrm{i})=0
\end{aligned}
$$

Where, $F=-E I-\left(e_{0} a\right)^{2} N_{t 1}+\left(e_{0} a\right)^{2} M U^{2}$ and $H=m\left(e_{0} a\right)^{2}(i \omega)^{2} L^{2}+\left(e_{0} a\right)^{2} K \cdot L^{2}+\left(e_{0} a\right)^{2} M(i \omega)^{2} L^{2}$ We can calculate $\mathrm{W}(\mathrm{i})$, up to $n$ terms from the eq.-14, by assuming $\mathrm{W}(1)=\mathrm{C}_{1}, \mathrm{~W}(3)=\mathrm{C}_{2}$ and it will be substituted in eq.-15 and 16 and by solving these equations for non-trivial solution, natural frequency 
$(\omega)$ of the CNT can be calculated. The accuracy of natural frequency increases with increase in the value of $n$ (number of iterations)and saturates at a maximum $n$ value, i.e $\mathrm{n}=\mathrm{Nmax}$.

\section{Fixed at Both Ends}

For the SWCNT supported by clamped at both the ends, the boundary conditions defined as $w(0)=0, w^{\prime}(0)=0, w(L)=0, w^{\prime}(L)=0$

By using Differential Transformation these can be written as:

$W(0)=0, W(1)=0$

$\sum_{i=0}^{N} W(\mathrm{i})=0, \sum_{i=0}^{N} i . W(\mathrm{i})=0$

We can calculate $\mathrm{W}$ (i), up to $n$ terms from the eq.-14, by assuming $\mathrm{W}(2)=\mathrm{C}_{1}, \mathrm{~W}(3)=\mathrm{C}_{2}$ and it will be substituted in eqs.-18 and 20 and by solving these equations for non-trivial solution, natural frequency $(\omega)$ of the CNT can be calculated.

\section{Validation}

\section{RESULTS AND DISCUSSION}

In Fig.-2 and Fig.-3, validation of the results has been carried out with the results available in the literature ${ }^{22}$ the outer radius of the nanotube is Rout $=3.5 \mathrm{~nm}$ and thickness of the nanotube $\mathrm{h}=0.34 \mathrm{~nm}$. The mass density of single-walled carbon nanotube is $2.3 \mathrm{~g} / \mathrm{cm}^{3}$ with Young's modulus E of $1 \mathrm{TPa}$. The density of water is $1 \mathrm{~g} / \mathrm{cm}^{3}$, aspect ratio $\mathrm{L} /(2 \mathrm{Rout})=100$, nonlocal parameter $\mathrm{e}_{0} \mathrm{a} / \mathrm{L}$ is taken from 0 to 0.05 and Winkler constant $\mathrm{K}$ is considered from 0 to $0.1 \mathrm{MPa}$.

In the present study, we consider two cases of temperature region, low and high. The coefficient of thermal expansion $\propto_{\mathrm{x}}=-1.6 \times 10^{-6} \mathrm{~K}^{-1}$ and $1.1 \times 10^{-6} \mathrm{~K}^{-1}$ for low or room temperature and high-temperature regions, respectively are considered. The Poisson's ratio is considered as 0.3.

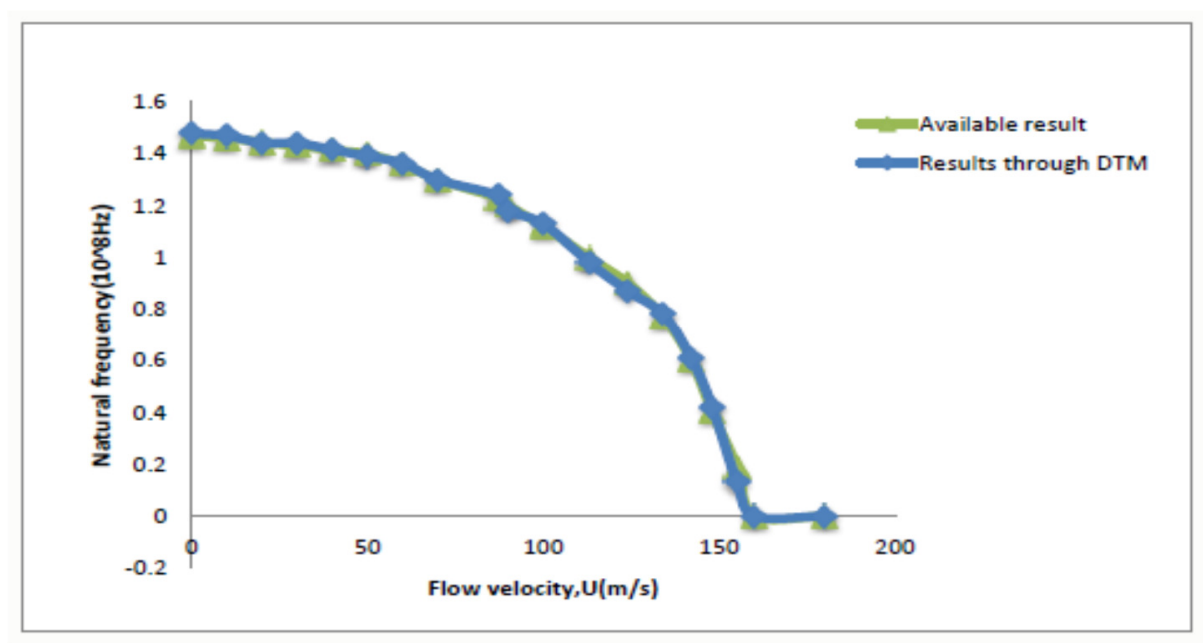

Fig.-2: Natural frequency v/s flow velocity (At $\mathrm{T}=25 \mathrm{~K}, \mathrm{e}_{0} \mathrm{a} / \mathrm{L}=0.05, \mathrm{~K}=0 \mathrm{MPa}$ )

Above figure is drawn between the natural frequency (y-axis) of the beam and fluid flow velocity(x-axis) entering through it in a high-temperature region. We observe that the natural frequency decreases with increase in flow velocity also, the results or data obtained here are very close to the available results of TChang et $\mathrm{al}^{22}$.

The nanotube becomes more flexible and the natural frequencies get reduce with increase in flow velocity. The fundamental natural frequency becomes zero and the nanotube becomes unstable. When the flow velocity exceeds a certain value, this corresponds to the instability of the single walled carbon 
nanotube and the flow velocity which produces the zero natural frequency defined as the critical flow velocity of the system. Because this velocity gives a perfect damping effect to the vibration.

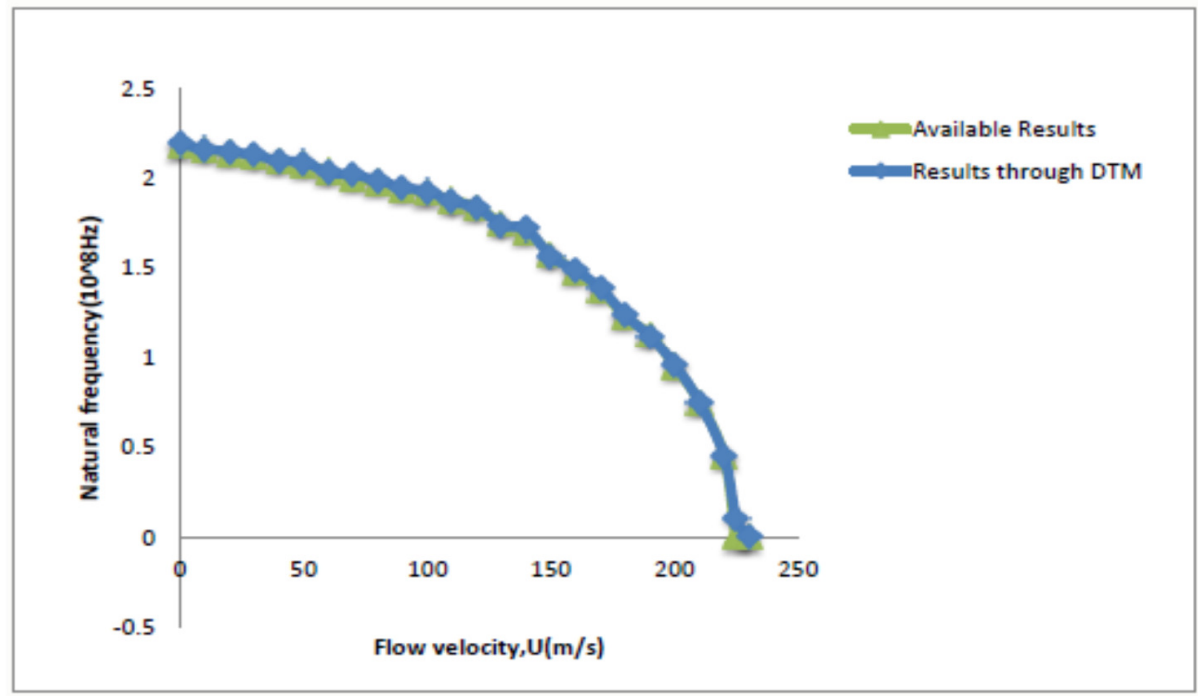

Fig.-3: Natural frequency v/s flow velocity (At $\mathrm{T}=15 \mathrm{~K}, \mathrm{e}_{0} \mathrm{a} / \mathrm{L}=0.05, \mathrm{~K}=0 \mathrm{MPa}$ )

Above figure is drawn in a low-temperature region by taking same boundary condition on the same axes as in Fig.-2. We observe that the natural frequency decreases with increase in flow velocity.

After the validation of the results, we have observed that the application of the differential transformation method gives the values very close to the available results in the literature ${ }^{22}$.

\section{Effect of the Nonlocalparameter}

Below figure is drawn in a high-temperature region. We observe that the variation of $\mathrm{e}_{0} \mathrm{a} / \mathrm{L}$ from 0 to 0.05 have a significant effect on the natural frequency at zero flow velocity but does not affect so much the critical flow velocity.

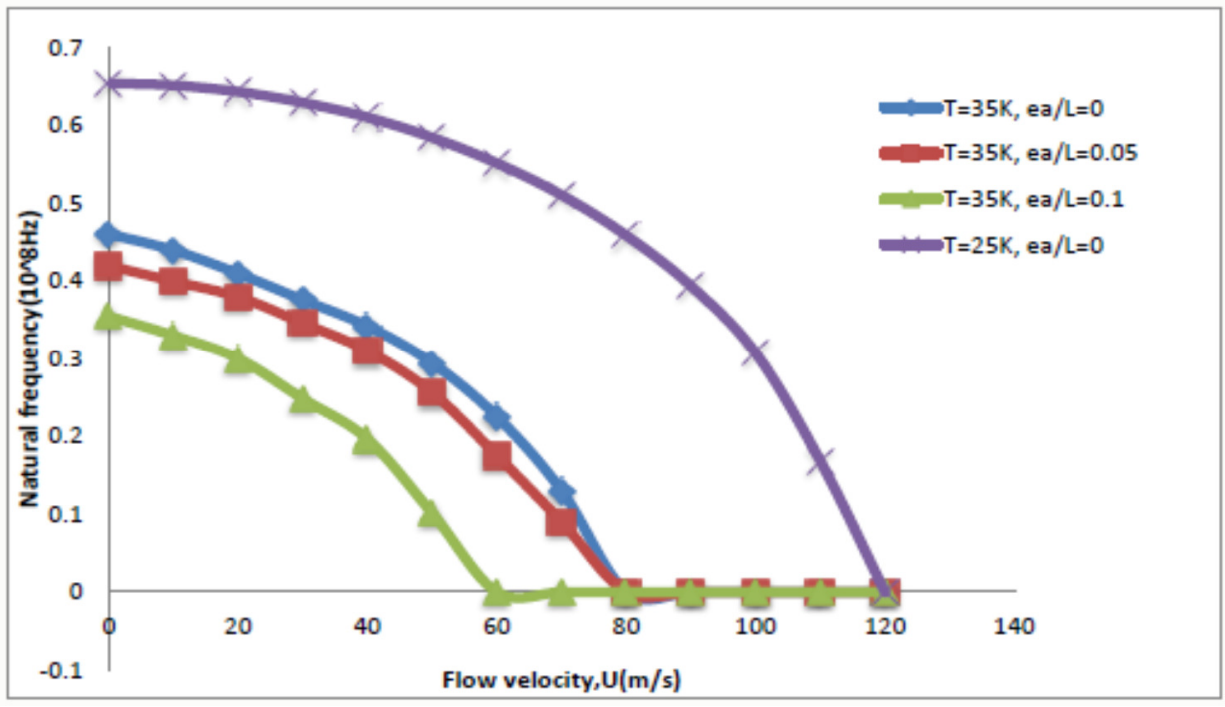

Fig.-4: Natural frequency v/s flow velocity (At K=0 MPa, Simply supported B.C)

When keeping $\mathrm{e}_{0} \mathrm{a} / \mathrm{L}=0$ the nonlocal beam theory reduces to a local beam theory. A curve is also drawn at $\mathrm{T}=25 \mathrm{~K}$ and with $\mathrm{e}_{0} \mathrm{a} / \mathrm{L}=0$, which corresponds to a local beam theory. We clearly observe that from local 
beam theory that the reduction of the natural frequencies and critical flow velocities happens when the nonlocal parameter is introduced.

A decrease in the natural frequency was related to the transverse force for following reasons:

(1) The change of curvature of nanotubes.

(2) The surface stress because of the nonlocal atom to atom interaction.

\section{Effects of the Winkler elastic constant}

Winkler type elastic medium gives normal pressure to the beam, which tightens it and hence introduces some stiffness to the beam against deflection. We are considering the range of variation for the $\mathrm{K}$ from 0 to $0.1 \mathrm{MPa}$.

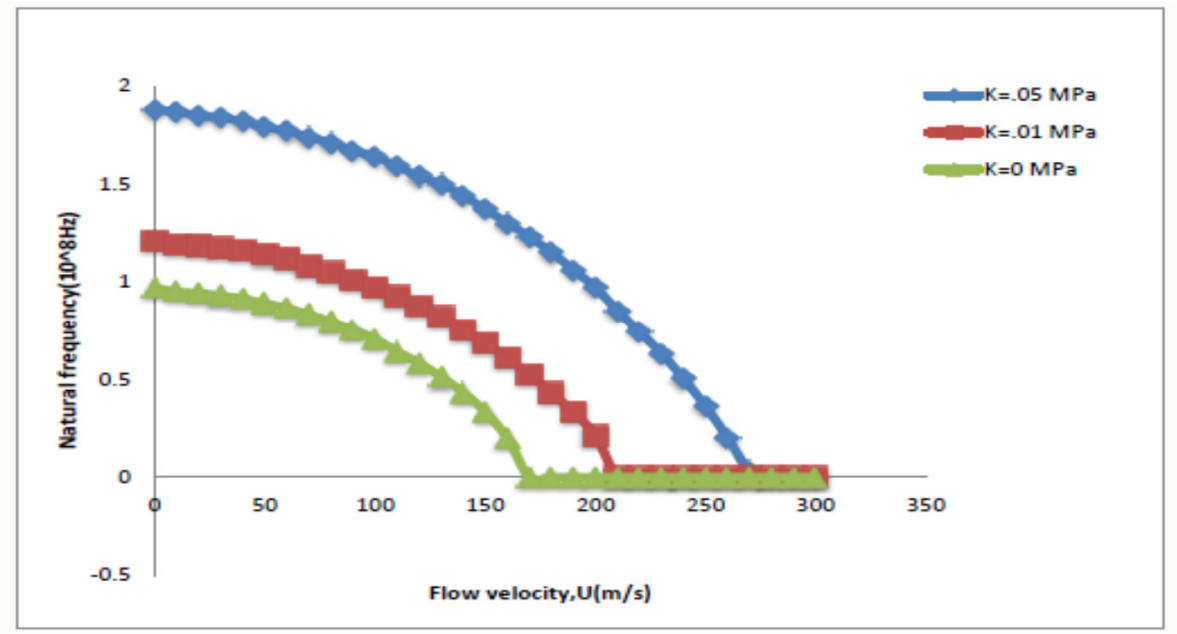

Fig.-6:Natural frequency v/s flow velocity (At $\mathrm{T}=0$ and $\mathrm{e}_{0} \mathrm{a} / \mathrm{L}=0.05$, simply supported B.C).

In above figure, the variation in Winkler constant is shown in the low-temperature region. We observe that the increase in Winkler's constant tends to increase in natural frequency as well as increase the critical flow velocity. At zero flow velocity natural frequency varies from $1 \times 10^{8} \mathrm{~Hz}$ to $1.87 \times 10^{8} \mathrm{~Hz}$ and critical flow velocity varies from $165 \mathrm{~m} / \mathrm{s}$ to $275 \mathrm{~m} / \mathrm{s}$. Although this graph is drawn under the lowtemperature region, it can also be found that Winkler foundation increases the natural frequency and critical flow velocity in a high-temperature region.

\section{Effects of the magnetic field}

Here we consider the Lorentz magnetic force given by Maxwell's ${ }^{14}$ relations within the range of nonlocal elasticity theory in the transverse direction. Frequency ratio is taken as the ratio of nonlocal frequency w.r.to local frequency of the beam. The value of magnetic permeability $\mu$ is taken as $4 \pi \times 10^{-7} \mathrm{H} . \mathrm{m}^{-1}$ or N. $A^{-2}$.

We observe that the value of frequency ratio is decreasing with the increase of the value of small scale coefficient $\mathrm{e}_{0} \mathrm{a} / \mathrm{L}$. This means that the fundamental frequencies of a single-walled carbon nanotube including the small-scale effects are less than the fundamental frequencies of the same beam excluding the small scale effects. This is due to the surface stress inaccount of the nonlocal atom to atom interaction. Incorporate

Nonlocal effect of the single-walled carbon nanotube is dampened by the presence of a longitudinal magnetic field (Fig.-7). It is observed in the present analysis that there is an increase in the fundamental frequency of the beam due to the presence of the magnetic field. This can be due to the coupling effect of vibrating nanotube and the magnetic field. As we increase the longitudinal field vector or strength of the field Hx, it suppresses the nonlocal behavior of the single-walled carbon nanotube. The present work can 
be extended to study the vibration of Single-walled carbon nanotube under the effect of transverse and inclined magnetic fields.

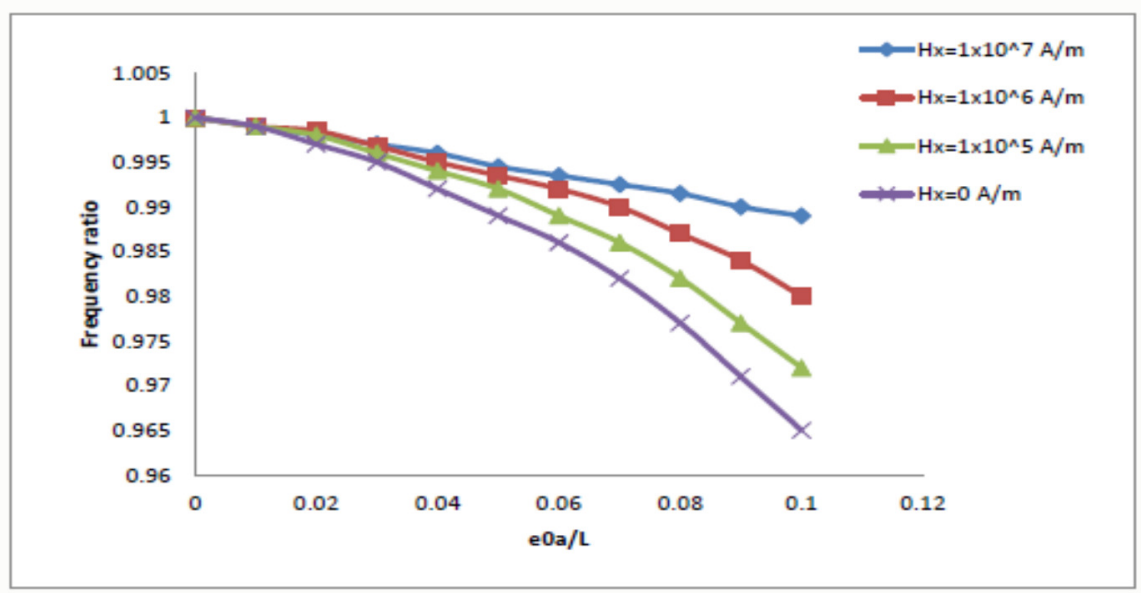

Fig.-7: Frequency ratio vs nonlocal parameter for simply supported boundary condition.

\section{CONCLUSION}

The present work gives an analytical method for studying the effects of temperature change, the nonlocal parameter, Winkler medium, and magnetic field on the natural frequency of single-walled carbon nanotube conveying fluid for various boundary conditions. Using the thermo- elastic mechanics theory and nonlocal elasticity, a Bernoulli-Euler beam model is created for the dynamic study of an SWCNT embedded in an elastic medium with magnetic effects. Differential transform method which is called semi-analytical numerical technique is used to solve governing differential equations and boundary conditions in a simple and accurate way. Various results are presented on the variation of the vibration frequency of single-walled carbon nanotube with critical flow velocity for various parameters. It is observed that at low or room temperature, natural frequency and critical flow velocity for the singlewalled carbon nanotube increase with the increase of the change of temperature, whereas in hightemperature range the natural frequency and critical flow velocity for the single-walled carbon nanotube decrease with the increase of the temperature change.

From this analysis, it can be seen that the solutions obtained for a single-walled carbon nanotube can be helpful in investigating more complicated nanotube structures with nonlocal effects and design of nanotubes in a transverse and inclined magnetic fields which may be used in space elevator for aerospace applications in future.

1. S. Iijima, Nature, 58, 354 (1991).

\section{REFERENCES}

2. X. Wang, Q. Li, J. Xie, Z. Jin, J. Wang, Y. Li, \& S. Fan, Nano Letters, 9(9), 3137(2009).

3. E. T. Thostenson, Z. Ren, \& T. W. Chou, Composites science and technology, 61(13), 1899(2001).

4. A.C. Eringen, J. Appl. Phys. 54, 4703(1983).

5. D. Qian, G.J. Wagner, W.K. Liu, M.F. Yu, R.S. Ruoff, Appl. Mech. Rev. 55, 495(2002).

6. E. Pop, D. A. Mann, K. E. Goodson, \& H. Dai, Journal of Applied Physics, 101(9), 093710 (2007).

7. E. Pop, D. Mann, Q. Wang, K. Goodson, \& H. Dai, Nano Letters,6(1), 96-100 (2006).

8. M. J. Treacy, T. W. Ebbesen, \& J. M. Gibson, Nature, 381(6584), 678(1996).

9. L. J. Sudak, Journal of Applied Physics, 94(11), 7281(2003).

10. Q. Wang, \& V. K. Varadan, Smart Materials and Structures, 14(1), 281(2005).

11. B. I. Yakobson, C. J. Brabec, \& J. Bernholc, Physical review letters, 76(14), 2511(1996).

12. A. Sears, \& R. C. Batra, Physical Review B, 73(8), 085410(2006).

13. J. N. Reddy, J. N., International Journal of Engineering Science, 45(2), 288-307(2007).

14. B. Ravi Kumar, International Journal of ChemTech Research,10(3), 339(2017). 
RASĀYAN J. Chem.

Vol. 10 | No. 2 |643 -651 | April - June | 2017

15. T. Murmu, S.C. Pradhan, Comput. Mater. Sci.47, 721(2010).

16. J. K. Zhou, Huazhong Science \& Technology University Press, China (1986).

17. C. O. K. Chen, \& S. H. Ho, Applied Mathematics and Computation, 106(2), 171(1999).

18. A. Arikoglu, \& I. Ozkol, Applied Mathematics and Computation, 168(2), 1145-1158(2005).

19. R. Kumar, \& S. Deol, Nonlocal Buckling Analysis of Single-Walled Carbon Nanotube Using Differential Transform Method (DTM). International J. Sci. Res., 5, (2016).

20. J. N. Reddy, \& S. D. Pang, Journal of Applied Physics, 103(2), 023511(2008).

21. S. C. Pradhan, \& G. K. Reddy, Computational Materials Science, 50(3), 1052-1056(2011).

22. T. P. Chang, T. P, Applied Mathematical Modelling, 36(5), 1964-1973(2012).

23. V. S. Angulakshmi, S. Karthikeyan, et.al., Rasayan Journal of Chemistry,8(1), 1-7(2015).

24. K. Sivaram, et al., Rasayan Journal of Chemistry, 10(1),16-24 (2017).

25. B. Ravi Kumar and K. Palaksha Reddy, Rasayan Journal of Chemistry,10(2), 481-487(2017).

[RJC-1669/2017 